Randomised controlled trial

\section{Morphine is not superior to ibuprofen for managing children's pain following minor orthopedic surgery}

10.1136/eb-2017-102855

Check for updates

Cornelius Botha Groenewald

Department of Anesthesiology and Pain Medicine, University of Washington, Seattle Children's Hospital, Seattle, Washington, USA

Correspondence to: Dr Cornelius Botha Groenewald, Department of Anesthesiology and Pain Medicine, University of Washington, Seattle Children's Hospital, Seattle, WA 98105, USA; cornelius.groenewald@ seattlechildrens.org

Commentary on: Poonai N, Datoo N, Ali S, et al. Oral morphine versus ibuprofen administered at home for postoperative orthopaedic pain in children: a randomised controlled trial. CMAJ 2017;189:E1252-8.

\section{Implications for practice and research}

- Nurses should encourage parents to use ibuprofen along with paracetamol as the first-line treatment for their children's postoperative pain at home.

- Future research is needed to determine the added benefit versus risk of using morphine alongside ibuprofen for postoperative pain control.

\section{Context}

Morphine and other opioids such as oxycodone and hydrocodone increasingly serve as the cornerstone of children's postoperative pain treatment. Yet, opioid use may result in a number of complications, including respiratory depression, nausea, constipation and an increased risk for opioid misuse later in life. Given the associated risks, it is important that evidence support the use of opioids over other reliable analgesics such as ibuprofen and paracetamol. However, such evidence is lacking in paediatric outpatient surgery. To fill this gap in knowledge, Poonai et al set out to determine whether oral morphine is more effective than ibuprofen in controlling children's pain at home following surgery. ${ }^{1}$

\section{Methods}

The primary aim was to determine whether oral morphine was superior to oral ibuprofen for controlling children's postoperative pain at home. Participants were 154 children 5-17years of age (mean age=12.4years) undergoing outpatient orthopaedic surgery. Researchers performed an intention to treat randomised controlled trial, which meant that every subject was analysed according to their randomised treatment assignment. Intentionto-treat analysis reduced bias introduced by participants dropping out of the study or being non-compliant with their treatment allocation (ie, took both ibuprofen and morphine). In total, 77 patients were randomised to each medication. Participants rated pain using the Faces Pain Scale-Revised. The six-item Faces Pain Scale-Revised is a validated measure of children's postoperative pain and is scored from 0 (no pain) to 10 (maximum pain). The primary outcome was the decrease in self-reported pain score following the first at home dose of pain medication.

\section{Findings}

On average, both morphine and ibuprofen provided clinically meaningful reduction of pain scores by one point (from 3 to 2; $p=0.2$ ) on the Faces Pain Scale-Revised. Thus, researchers concluded that oral morphine was not superior to oral ibuprofen for controlling children's postoperative pain at home. An important secondary finding was that morphine was associated with more side effects compared with ibuprofen.

\section{Commentary}

Opioids are considered standard of care for postoperative pain management and $>60 \%$ of children undergoing outpatient procedures fill an opioid prescription in the USA. ${ }^{2}$ Nevertheless, opioid use by children is directly associated with increased risk of harms such as respiratory depression, accidental overdose and future opioid misuse. ${ }^{3}$ Since the goals of perioperative pain management are to minimise suffering and avoid harmful side effects, it is surprising that data comparing opioids to lower risk alternatives such as ibuprofen is limited.

The authors suggest that ibuprofen is a better first-line analgesic after children's outpatient surgery. The study was appropriately designed to make this strong conclusion: participants were randomised, both observers and patients were adequately blinded, data were analysed in an intention-totreat analysis and the primary outcome was measured using a validated instrument. Thus, readers of this article can be confident in the findings. Furthermore, findings from this study will be used by stakeholders, including clinicians, health policymakers and parents, in efforts to reduce use of opioid pain medications by children. Specifically, in the USA, there is now a strong drive to limit opioid prescribing to children and to treat acute pain with non-opioid medications. ${ }^{4}$

However, we should also consider two other important limitations. First, multimodal analgesia is superior to single modality use for postoperative analgesia. Thus, future studies should determine whether morphine in combination with ibuprofen provides superior pain relief as compared with ibuprofen alone. Second, a limitation of all randomised trials is that study participants are chosen using narrow criteria which limits generalisability of findings to the larger childhood population.

In conclusion, this is a well designed and executed study which found that morphine was not superior to ibuprofen for relieving children's pain following minor orthopaedic surgery. Parents should use ibuprofen and paracetamol as first-line treatment for their children's postoperative pain at home.

\section{Competing interests None declared.}

Provenance and peer review Commissioned; internally peer reviewed.

- Article author(s) (or their employer(s) unless otherwise stated in the text of the article) 2018. All rights reserved. No commercial use is permitted unless otherwise expressly granted.

\section{References}

1 Poonai N, Datoo N, Ali S, et al. Oral morphine versus ibuprofen administered at home for postoperative orthopedic pain in children: a randomized controlled trial. CMAJ 2017;189:E1252-8.

2 Harbaugh CM, Lee JS, Hu HM, et al. Persistent opioid use among pediatric patients after surgery. Pediatrics 2018;141:e20172439.

3 Miech R, Johnston L, 0'Malley PM, et al. Prescription opioids in adolescence and future opioid misuse. Pediatrics 2015;136:e1169-77.

4 United States Department of Health \& Human Services. Office of Adolescent Health. Opioids and adolescents. https://www.hhs.gov/ash/oah/adolescentdevelopment/substance-use/drugs/opioids/index.html\#prevention (accessed Jan 2018). 